\title{
What is a "Lament," Really?: A Commentary on Nicholas Shea's "Descending Bass Schemata and Negative Emotion in Western Song"
}

\author{
BRYN HUGHES \\ University of Lethbridge [1]
}

\begin{abstract}
This commentary focuses on Shea (2019) and its relationship to much of the literature on popular and rock music. The commentary offers some methodological considerations on the construction of corpora for this type of analysis. The commentary questions the operational definition of 'lament,' and laud's Shea's work in attempting to create a more thorough, objective definition. Ultimately, the commentary concludes that, while Shea's approach is worthwhile, a much larger corpus must be generated in order to draw meaningful conclusions from it.
\end{abstract}

Submitted 2019 September 26; accepted 2019 November 2.

Published 2020 July 6; https://doi.org/10.18061/emr.v14i3-4.7570

KEYWORDS: commentary, corpus study, lament, emotion

NICHOLAS Shea's article "Descending Bass Schemata and Negative Emotion in Western Song" investigates the widely held assumption by music scholars that the lament bass topic is associated with negative emotion in Western music and is perceived as such by Western listeners. Music theorists have produced compelling analyses of Western classical music that reveal a relationship between a descending bass and text that expresses negative emotions, such as lament, sadness, melancholy, wistfulness, and nostalgia. Very little of music theory scholarship has engaged the lament topic as it pertains to popular music; however, Shea's work delves deep into this repertoire, making it a welcome and needed investigation of whether our assumptions about Western European classical music have a broader applicability. In addition, Shea's methodology employs both corpus analysis and a behavioural study, which allows for an empirical perspective that nicely supplements those put forth by music analysts.

\section{DEFINING 'LAMENT'}

Shea combs through the scholarly literature in an attempt to find an operational definition of "lament." $\mathrm{He}$ appropriately draws a connection between the "sigh' vocalization and pitch descent; however, this connection is not made explicitly to the bass voice of a multiphonic texture. He cites Kodály's discussion of Hungarian laments and their characteristic descending lines; however, it is unclear whether these lines are meant to be heard specifically in the bass voice. Both Schmalfeldt (2001) and Caplin (2014) link the lament to the bass voices and mention its frequent use as a text-painting device. Schmalfeldt's analysis of Purcell's "When I am laid in earth," from Dido and Aeneas, suggests that numerous elements may contribute to the sense of lament. Caplin is more explicit about the lament topic being tied to the bass; however, Caplin does not commit to linking the lament to sadness. Likewise, Christopher Doll (2017) identifies laments in popular music, but does not associate these root progressions with the lyrical content they accompany. Caplin and Doll both limit the lament topic to the minor mode, but Shea aptly points out major-mode examples from both the Common Practice and popular music repertoire that might be considered laments. In doing so, Shea expands the emotional association of the lament beyond sadness to include nostalgia, fear, and anger. Considering all of this evidence from the music theory literature, the definition of 'lament' as a musical topic seems tenuous. One could categorize a lament as a descending musical gesture that is: in the bass (or not), in minor mode (or not), that conveys a negative emotion such as sadness (or not). Finally, Shea mentions that pitch descent may in fact be a universal tendency in Western music, which broadens the potential definition so much that it becomes no longer useful. Taken all together, the definitions of "lament 
bass" in the music theory literature suggest categorical boundaries that are too permissive to be useful. Shea's computational method is a valiant effort to rectify this problem.

\section{EXPERIMENT 1}

Shea's first study analyzes two corpora in an attempt to find associations with descending bass lines and lyrics that connote sadness. He chooses a collection of Bach cantatas (ca. 1720-50) as a corpus representative of common practice repertoire; this is contrasted with the McGill Billboard Hot 100 database, which he claims is the "most stylistically diverse and historically expansive" corpus available for popular music. Shea uses computational analysis methods to cross-reference songs containing descending bass lines with songs that include a low "tone" rating per a quantitative psychometric text analysis. Shea predicts that: "songs with descending bass lines [will be] more closely associated with lyrics that convey negative emotions." However, he finds no significant difference in negative emotion values between songs with descending and non-descending bass lines in either corpus.

\section{FINDING LAMENTS IN THE CORPORA}

Investigating the Bach corpus, Shea first defines "descending bass" as a line that moves downward from the first through the fifth scale degree, allowing both diatonic and chromatic versions of this descent to count. Further, Shea includes descending bass lines that occur at any of three levels of metric hierarchy, allowing patterns in which the requisite scale degrees occur on every other note in addition to those in which the scale degrees unfold contiguously. He uses the same procedure with the Billboard corpus to find potential laments in popular music. In both cases, major- and minor-mode examples were considered. Importantly, only cantata movements with eight or more iterations of the bass pattern were used; however, it is unclear whether or not this limitation was applied to the popular music corpus. The results of these procedures yielded only 95 of the 760 songs included in the Billboard corpus.[2] Even before lyric analysis, the sample of songs from this corpus is remarkably small.

Shea analyzes the lyric content of both corpora using a psychometric text analysis tool called the Linguistic Inquiry and Word Count database (LIWC), which produces a "tone value" based on word content and function. In these analyses he encounters another problem: The tool depends on word counts for its output, and the Bach cantatas contain considerably fewer words than do the songs from the Billboard corpus. Shea's solution is to use only the cantatas that include 39 words or more (the median word count for the corpus), effectively cutting the size of the corpus in half. With these caveats, Shea ends up with 31 descending bass lines from the Bach corpus to accompany the 95 from the Billboard corpus. With this relatively small sample, Shea's analysis reveals no significant difference between the emotional content of songs with descending bass lines when compared to those with non-descending bass lines. Per his analysis, one might conclude that the notion of the lament topic connoting sadness and other negative emotions is unsupported by the repertoire.

Shea rightly speculates whether distinguishing between major- and minor-mode songs/cantatas might change the results; however, he is unable to follow this line of inquiry due to the degree to which this would shrink the already small sample. Of the Bach cantatas, among those that include 39 or more words, only 13 are in minor mode and also feature a descending bass pattern. This highlights two flaws: The corpus needs to be bigger, and the methodology needs to be more inclusive. Although it would require a substantial amount of work, a larger corpus would allow for more definitive claims to be made about the emotional content of songs with descending bass patterns. Alternatively, a method of text analysis that doesn't depend on word count might allow for more of the Bach corpus to be included. Both of these approaches might allow for a direct comparison of major- and minor-mode samples, which would more closely align with the speculations made by Caplin and Doll that the lament topic is exclusively a minormode phenomenon.

The Billboard corpus puts forward a different problem in determining the impact of the minor mode on this analysis: Popular music tends to be less clearly in a major or minor mode, overall. Nevertheless, Shea manually analyzed the songs from the Billboard corpus with descending bass lines and cross-validated his analyses with one provided by Spotify metadata. Using only the songs that were determined to be in minor mode per the cross-validated analyses yielded just 30 songs - a sample too small to be useful. Again, the problem lies with the corpus itself: if the musical features we deem most important are too much of a constraint, then the corpus needs to be bigger. One suggestion would be to look at songs 
from a repertoire that we know to be more closely aligned to the major/minor system. Walter Everett (Everett, 2004) points out that 1950s and 1960s rock does exactly this; perhaps building a corpus of those songs exclusively might produce a larger sample overall.

\section{EXPERIMENT 2}

In a follow-up behavioural study, Shea tests whether songs with descending bass lines are judged to have more acoustic features that are associated with sadness. Participants listened to a sample of songs from the Billboard corpus categorized as having either a descending or non-descending bass line and rated the presence of musical features related to sadness. The study borrows this paradigm from previous research by Juslin \& Lukka (Juslin \& Laukka, 2003), and Huron et al. (Huron, Anderson, \& Shanahan, 2014). Shea hypothesizes that the presence of a descending bass line would more strongly predict ratings for loudness, tempo, interval size, articulation, and timbre than those with non-descending bass lines. The results revealed that only tempo, and articulation served as significant predictors. Notably, listeners' perceptions of the presence of small intervals did not significantly predict whether the song had a descending or nondescending bass line. He concludes that the presence of small intervals (and by extension, a descending bass line) is not something that is particularly salient to listeners, and thus has minimal impact on a listener's perception of the song as having negative emotional content. Shea proposes that harmonic content (as related to the descending bass pattern) is likely secondary to other musical features, such as pace and articulation, in "conveying emotional affect."

As noted above, the sample size made it difficult to find examples of songs in the corpus that were feature-laden. When scrutinizing the list of songs included in the descending bass block of trials, it becomes easy to see why participants may not have differentiated them from the control block. Many of the songs determined by Shea's computational analysis to have a descending bass only did so in the most fleeting sense. After listening through each of these songs in their entirety, "Tonight, Tonight, Tonight," "Sail on Sailor," and "Bluebirds Over the Mountains" have no obvious descending bass line; "Sanctify Yourself," "Town Without Pity," "Silver Threads and Golden Moons," and "Black Cars" feature descending bass patterns only in very brief moments, and in some cases those moments spill over sectional boundaries. The remaining stimuli have clearer descending bass lines, but as noted above, several are in the major mode, and the scale degrees necessary for the descending pattern may only appear non-contiguously. As it was mentioned previously, the Bach cantatas were only considered having descending bass lines if eight or more iterations of that line occurred in the movement. It is not clear that this caveat was placed on the stimuli drawn from the Billboard corpus - it would likely have excluded several of the examples noted above. Further, it's not clear that the lyrics determined by LIWC to have a negative tone value aligned at all with the moments in which the descending bass lines occurred in these songs.

Like Experiment 1, it's possible that this study could have produced different results with a more controlled sample of descending bass line stimuli. This could be achieved by using only songs that are firmly in the minor-mode and feature a contiguous stepwise descending bass line, drawn either from a more focused corpus, or with novel stimuli composed specifically for the study.

\section{CONCLUSIONS}

Shea's work attempts to deconstruct the lament topic: He separates the musical from the extramusical features (specifically the text) in order to discern whether or not the descending line itself connotes any emotional meaning. This kind of empirical inquiry testing the long-held assumptions of music theorists is important and commendable. Shea goes further, though, testing whether these assumptions extend to nonminor-mode bass lines, and whether they extend beyond the common practice repertoire. His pairing of the Linguistic Inquiry and Word Count database tool for text analysis with music corpus analysis is an exciting methodology for future work in this area. Finally, his attempt at comparing two corpora (representing two distinct musical styles), in this regard contributes to ongoing research involving the intersection of music theory as it applies to both common-practice and popular music. My criticisms of this article are not with its intent or its approach, but rather with some details in its execution. I hope that this research continues into the future, as I believe it has the potential to provide a valuable contribution to our disciplines. 


\section{ACKNOWLEDGEMENTS}

This article was copyedited by Niels Chr. Hansen and layout edited by Diana Kayser.

\section{NOTES}

[1] Correspondence can be addressed to: Bryn Hughes, Assistant Professor of Music Theory, University of Lethbridge, 4401 University Dr W, Lethbridge, AB T1K 3M4, Canada. bryn.hughes@uleth.ca

[2] Shea does not report the number of cantatas this procedure yields when analyzing the Bach corpus; he instead prunes this corpus further with regard to its lyric content and reports that tally instead.

\section{REFERENCES}

Caplin, William. (2014). "Topics and Formal Functions: The Case of the Lament," in The Oxford Handbook of Topic Theory, edited by Danuta Mirka, 415-452. New York: Oxford University Press. https://doi.org/10.1093/oxfordhb/9780199841578.013.0016

Doll, C. (2017). Hearing Harmony: Toward a Tonal Theory for the Rock Era. Ann Arbor: University of Michigan Press. https://doi.org/10.3998/mpub.3079295

Everett, W. (2004). Making Sense of Rock's Tonal Systems. Music Theory Online, 10(4). Retrieved from http://www.mtosmt.org/issues/mto.04.10.4/mto.04.10.4.w_everett.html

Huron, D., Anderson, N., \& Shanahan, D. (2014). "You Can't Play a Sad Song on the Banjo:" Acoustic Factors in the Judgment of Instrument Capacity to Convey Sadness. Empirical Musicology Review, 9(1), 29-41. https://doi.org/10.18061/emr.v9i1.4085

Juslin, P. N., \& Laukka, P. (2003). Communication of emotions in vocal expression and music performance: Different channels, same code? Psychological Bulletin, 129(5), 770-814. https://doi.org/10.1037/0033-2909.129.5.770

Schmalfeldt, J. (2001). In Search of Dido. The Journal of Musicology, 18(4), 584-615. https://doi.org/10.1525/jm.2001.18.4.584 